Najeeb Alam Khan*, Fatima Riaz, and Oyoon Abdul Razzaq

\title{
A comparison between numerical methods for solving Fuzzy fractional differential equations
}

\begin{abstract}
In this paper, numerical simulation of linear and nonlinear fuzzy initial value problems of fractional order has been done. A modification in the improved Euler's method for fuzzy fractional order is proposed and utilized. Also, the two component homotopy perturbation method also known as a modified homotopy perturbation method (MHPM) is employed to solve the considered problems. The results obtained from the considered techniques are compared. These methods are illustrated by solving several examples. Efficiency and exactness of results worked out from MHPM is examined from the tables and comparatively it is found that MHPM is more accurate and closer to the exact solutions than the existing methods.
\end{abstract}

Keywords: Fractional order, Fuzzy initial value problem (FIVP), perturbation method, Fuzzy differential equations, improved fractional Euler's method (IFEM)

DOI 10.1515/nleng-2013-0029

Received December 17, 2013; accepted April 22, 2014.

\section{Introduction}

Fuzzy differential equations serve as mathematical models for many exciting real-world problems. Not only in science and technology but also in diverse fields such as population models, civil engineering, and modeling hydraulics.

Unfortunately every time uncertainty can intervene with real world problems while modeling differential equations. Fuzzy set theory is a powerful tool to overcome the problems of uncertainty that arise from deficient data, measurement errors or when determining initial conditions for differential equations. Initially the concept of fuzzy derivative was first introduced by Chang et al. [1], fol-

\footnotetext{
*Corresponding Author: Najeeb Alam Khan: Department of Mathematical Sciences, University of Karachi, Karachi 75270, Pakistan, E-mail: njbalam@yahoo.com

Fatima Riaz: Department of Mathematical Sciences, University of Karachi, Karachi 75270, Pakistan

Oyoon Abdul Razzaq: Alhamd Islamic University, Quetta 87300, Pakistan
}

lowed by Dubois et al. [2], who defined and used the extension principle in their approach. The derivative for fuzzy valued mappings was developed by Puri et al. [3], who generalized and extended the concept of Hukuhara differentiability for set valued mappings to the class of fuzzy mappings. Subsequently, using the Hukuhara derivative, Kaleva [4, 5], Seikkala [6] and others, started to develop the theory for Fuzzy differential equations. The numerical methods to solve fuzzy differential equations were introduced in [7-11].

In the last few decades, scientists and applied mathematicians have found fractional differential equations constructive in various fields such as rheology, quantitative biology, electrochemistry, scattering theory, diffusion, transport theory, probability potential theory and elasticity. Many important physical phenomena in the engineering and science fields are frequently modeled by nonlinear differential equations. Such equations are often difficult or impossible to solve in a closed way. Nevertheless, analytical methods have gained significant importance to obtain approximate solutions of nonlinear fractional problems. The researchers proposed many methods to solve fractional order differential equations which serve as a key to study insight of the problems [12-17].

In recent years, the famous Euler's method introduced by Euler in 1728, has been considerably improved by increasing its order of correctness, and has been named as Improved Euler's method. This is a basic explicit method for numerical integration of ordinary differential equations with a given initial value and often serves as the basis to construct more complicated methods. This method has been found in the papers of Shorki [18] and Chakraverty et al. [19].

In this paper, a modification in fractional Euler's method (IFEM) is proposed which is further corrected by modified trapezoidal rule. The suggested approach is utilized and compared with the modification of homotopy perturbation method (MHPM) or two component homotopy perturbation method of fractional order to solve fuzzy differential equation. MHPM is modified combination of homotopy in topology and classic perturbation techniques. This method is advancement and modification of homotopy perturbation method (HPM). It was modified 
by scientists and physicians Chun [20], He [21], Yusufoglu [22] and Aminikhah and Hemmatnezhad [23]. Recently, it has been successfully applied to solve many types of linear and nonlinear problems in applied sciences. Its applications in nano-electronics are illustrated in the paper of Jafari et al. [24]. It has significant advantage as it provides an analytical approximation to a wide range of linear and nonlinear differential equations. The solutions retrieve from the adopted methods, recovers the previous results of Duraisamy and Usha [25] and improved Euler's type methods (Method 1 and Method 2) [19] as the special case for $\alpha=1$.

\section{Preliminaries}

In this section, several basic concepts of fuzzy number, Riemann-Liouville Integral and Caputo Fractional Derivative which were used in this article are recalled.

\section{Definition 2.1}

The Riemann-Liouville integral of order $\alpha>0$ for a function $f$ is defined by [26]

$$
J^{\alpha} f(x)=\int_{0}^{x} \frac{(x-t)^{\alpha-1}}{\Gamma(\alpha)} f(t) d t, \quad x>0
$$

\section{Definition 2.2}

Caputo's definition, which is a modification of the Riemann-Liouville definition, has the advantage of dealing properly with initial value problems in which the initial conditions are given in terms of the field variables and their integer order which is the case in most physical processes.

Lemma2.1 Let $f(x)$ be a crisp continuous function and $(\lceil\beta\rceil)$ - times differentiable in the independent variable $x$ over the interval of differentiation (integration) $[0, x]$. Then the following relation is hold [27]:

$$
{ }^{c} D^{\beta} f(x)={ }^{R L} D^{\beta}\left(f(x)-\sum_{k=0}^{\lfloor\beta\rfloor} \frac{x^{k}}{k !} f_{0}^{(k)}\right) \quad \beta \in(n-1, n),
$$

where, $f_{0}^{(k)}=\left.\frac{d^{k} f(x)}{d x^{k}}\right|_{x=0}$ and ${ }^{c} D^{\beta}$ is the Caputo derivative operator, $\lfloor\beta\rfloor$ and $\lceil\beta\rceil$ are just the values of $\beta$ rounded up and down to the nearest integer number respectively. ${ }^{R L} D^{\beta}$ is the more common Riemann-Liouville fractional derivative operator which can be defined as follows:

$$
{ }^{R L} D^{\beta} f(x)=\frac{1}{\Gamma(\lceil\beta\rceil-\beta)} \frac{d^{\lceil\beta\rceil}}{d x\lceil\beta\rceil} \int_{0}^{x} \frac{f(t)}{(x-t)^{1-\lceil\beta\rceil+\beta}} d t
$$

\section{Definition 2.3}

$$
\begin{aligned}
& \text { Let } \tilde{f}(x) \in C^{F}[a, b] \cap L^{F}[a, b], \\
& \qquad \tilde{G}(x)=\frac{1}{\Gamma(\lceil\beta\rceil-\beta)} \int_{0}^{x} \frac{\tilde{f}(t)-\sum_{k=0}^{\lfloor\beta\rfloor} \frac{t^{k} !}{k !} \bar{f}_{0}^{(k)}}{(x-t)^{1-\lceil\beta\rceil+\beta}} d t,
\end{aligned}
$$

$\tilde{H}\left(x_{0}\right)=\lim _{h \rightarrow 0^{+}} \frac{\tilde{G}\left(x_{0}+h\right) \Theta \tilde{G}\left(x_{0}\right)}{h}=\lim _{h \rightarrow 0^{+}} \frac{\tilde{G}\left(x_{0}\right) \Theta \tilde{G}\left(x_{0}-h\right)}{h}$,

$\tilde{L}\left(x_{0}\right)=\lim _{h \rightarrow 0^{+}} \frac{\tilde{G}\left(x_{0}\right) \Theta \tilde{G}\left(x_{0}+h\right)}{(-h)}=\lim _{h \rightarrow 0^{+}} \frac{\tilde{G}\left(x_{0}-h\right) \Theta \tilde{G}\left(x_{0}\right)}{(-h)}$.

$\tilde{f}(x)$ is the Caputo-type fuzzy differential function of order $0<\beta<2, \quad \beta \neq 1$ at $x_{0} \in(0, b)$, if there exist an element ${ }^{c} D^{\beta} f\left(x_{0}\right) \in C^{F}$ such that for all $0 \leq \alpha \leq 1$ and for $h>0$ sufficiently near zero, either.

${ }^{c} D^{\beta} f\left(x_{0}\right)=\lim _{h \rightarrow 0^{+}} \frac{\tilde{G}\left(x_{0}+h\right) \Theta \tilde{G}\left(x_{0}\right)}{h}=\lim _{h \rightarrow 0^{+}} \frac{\tilde{G}\left(x_{0}\right) \Theta \tilde{G}\left(x_{0}-h\right)}{h}$

${ }^{c} D^{\beta} f\left(x_{0}\right)=\lim _{h \rightarrow 0^{+}} \frac{\tilde{G}\left(x_{0}\right) \Theta \tilde{G}\left(x_{0}+h\right)}{(-h)}=\lim _{h \rightarrow 0^{+}} \frac{\tilde{G}\left(x_{0}-h\right) \Theta \tilde{G}\left(x_{0}\right)}{(-h)}$

For $0<\beta<1$ and either

${ }^{c} D^{\beta} f\left(x_{0}\right)=\lim _{h \rightarrow 0^{+}} \frac{\tilde{H}\left(x_{0}+h\right) \Theta \tilde{H}\left(x_{0}\right)}{h}=\lim _{h \rightarrow 0^{+}} \frac{\tilde{H}\left(x_{0}\right) \Theta \tilde{H}\left(x_{0}-h\right)}{h}$

${ }^{c} D^{\beta} f\left(x_{0}\right)=\lim _{h \rightarrow 0^{+}} \frac{\tilde{H}\left(x_{0}\right) \Theta \tilde{H}\left(x_{0}+h\right)}{(-h)}=\lim _{h \rightarrow 0^{+}} \frac{\tilde{H}\left(x_{0}-h\right) \Theta \tilde{H}\left(x_{0}\right)}{(-h)}$

${ }^{c} D^{\beta} f\left(x_{0}\right)=\lim _{h \rightarrow 0^{+}} \frac{\tilde{L}\left(x_{0}+h\right) \Theta \tilde{L}\left(x_{0}\right)}{h}=\lim _{h \rightarrow 0^{+}} \frac{\tilde{L}\left(x_{0}\right) \Theta \tilde{L}\left(x_{0}-h\right)}{h}$

${ }^{c} D^{\beta} f\left(x_{0}\right)=\lim _{h \rightarrow 0^{+}} \frac{\tilde{L}\left(x_{0}\right) \Theta \tilde{L}\left(x_{0}+h\right)}{(-h)}=\lim _{h \rightarrow 0^{+}} \frac{\tilde{L}\left(x_{0}-h\right) \Theta \tilde{L}\left(x_{0}\right)}{(-h)}$

$n \in N$

For $1<\beta<2$.

If the fuzzy valued function $\tilde{f}(x)$ is differentiable, as in Definition 3.1 cases (a, c, e), it is the Caputo-type differentiable in the first form and is denoted by ${ }^{c} D_{1}^{\beta} \tilde{f}(x),{ }^{c} D_{1,1}^{\beta} \tilde{f}(x)$ and ${ }^{c} D_{1,2}^{\beta} \tilde{f}(x)$ respectively. If $\tilde{f}(x)$ is differentiable, as in Definition 3.1 cases (b, d, f), it is the Caputo-type differentiable in the second form and is denoted by ${ }^{c} D_{2}^{\beta} \tilde{f}(x)$, ${ }^{c} D_{2,1}^{\beta} \tilde{f}(x)$ and ${ }^{c} D_{2,2}^{\beta} \tilde{f}(x)$ respectively.

Theorem 2.1: Let $f(x) \in C^{F}[a, b] \cap L^{F}[a, b]$ be a fuzzy valued function $[\tilde{f}(x)]^{r}=\left[\underline{f}^{r}(x), \bar{f}^{r}(x)\right]$, for $\alpha \in[0,1]$ and $x_{0} \in(0, b)$. Then 
(a) If $\tilde{f}(x)$ is a Caputo-type fuzzy fractional differentiable function in the first form, then for $0<\beta<1$

$$
\left[{ }^{c} D_{1}^{\beta} \tilde{f}(x)\right]^{\alpha}=\left[{ }^{c} D^{\beta} \underline{f}^{\alpha}(x),{ }^{c} D^{\beta} \bar{f}^{\alpha}(x)\right]
$$

(b) If $\tilde{f}(x)$ is a Caputo-type fuzzy fractional differentiable function in the second form, then for $0<\beta<1$

$$
\left[{ }^{c} D_{2}^{\beta} \tilde{f}(x)\right]^{\alpha}=\left[{ }^{c} D^{\beta} \underline{f}^{\alpha}(x),{ }^{c} D^{\beta} \bar{f}^{\alpha}(x)\right]
$$

(c) If ${ }^{c} D_{1}^{\beta} \tilde{f}(x)$ is a Caputo-type fuzzy fractional differentiable function in the first form, then for $1<\beta<2$

$$
\left[{ }^{c} D_{1,1}^{\beta} \tilde{f}(x)\right]^{\alpha}=\left[{ }^{c} D^{\beta} \underline{f}^{\alpha}(x),{ }^{c} D^{\beta} \bar{f}^{\alpha}(x)\right]
$$

(d) If ${ }^{c} D_{1}^{\beta} \tilde{f}(x)$ is a Caputo-type fuzzy fractional differentiable function in the second form, then for $1<\beta<2$

$$
\left[{ }^{c} D_{1,2}^{\beta} \tilde{f}(x)\right]^{\alpha}=\left[{ }^{c} D^{\beta} \underline{f}^{\alpha}(x),{ }^{c} D^{\beta} \bar{f}^{\alpha}(x)\right]
$$

(e) If ${ }^{c} D_{2}^{\beta} \tilde{f}(x)$ is a Caputo-type fuzzy fractional differentiable function in the first form, then for $1<\beta<2$

$$
\left[{ }^{c} D_{2,1}^{\beta} \tilde{f}(x)\right]^{\alpha}=\left[{ }^{c} D^{\beta} \underline{f}^{\alpha}(x),{ }^{c} D^{\beta} \bar{f}^{\alpha}(x)\right]
$$

(f) If ${ }^{c} D_{2}^{\beta} \tilde{f}(x)$ is a Caputo-type fuzzy fractional differentiable function in the second form, then for $1<\beta<2$

$$
\left[{ }^{c} D_{2,2}^{\beta} \tilde{f}(x)\right]^{\alpha}=\left[{ }^{c} D^{\beta} \underline{f}^{\alpha}(x),{ }^{c} D^{\beta} \bar{f}^{\alpha}(x)\right]
$$

Where

$$
\begin{gathered}
{ }^{c} D^{\beta} \underline{f}^{\alpha}\left(x_{0}\right)=\left[\frac{1}{\Gamma(\lceil\beta\rceil-\beta)} \int_{0}^{x} \frac{D^{\lceil\beta\rceil} f^{\alpha}(t)}{\left.(x-t)^{1-\lceil\beta\rceil+\beta} d t\right]}\right. \\
{ }^{c} D^{\beta} \bar{f}^{\alpha}\left(x_{0}\right)=\left[\frac{1}{\Gamma(\lceil\beta\rceil-\beta)} \int_{0}^{x} \frac{D^{\lceil\beta\rceil} \bar{f}^{\alpha}(t)}{(x-t)^{1-\lceil\beta\rceil+\beta}} d t\right] \\
D^{k} f(t)=\frac{d^{k} f(t)}{d t^{k}}
\end{gathered}
$$

\subsection{Fuzzy Number}

Fuzzy number is mapping $u=R \rightarrow[0,1]$ with the following properties:

(I) $u$ is upper semi-continuous.

(II) $u$ is fuzzy convex, i.e. $u(\lambda x+(1-\lambda) y) \geq \min \{u(x), u(y)\}$ for all $x, y \in R, \lambda \in[0,1]$

(III) $u$ is normal, i.e., $\exists x_{0} \in R$ for which $u\left(x_{0}\right)=1$

(IV) Supp $\overline{u=\{x \in R \mathrm{I}(u(x)>0\}}$ is the support of the $u$ and its closure $\operatorname{cl}(\operatorname{supp} u)$ is compact.

\subsection{Trapezoidal Fuzzy Number (TrFN)}

Let $A=(a, b, c, d), a<b<c<d$, be a fuzzy set on $R=$ $(-\infty, \infty)$. It is called a trapezoidal fuzzy number, if its membership function is

$$
\mu A(x)=\left\{\begin{array}{l}
\frac{x-a}{b-a}, \text { if } a \leq x \leq b \\
1, \quad \text { if } b \leq x \leq c \\
\frac{d-x}{d-c}, \quad \text { if } c \leq x \leq d \\
0, \quad \text { otherwise }
\end{array}\right.
$$

Though an $r$-cut approach trapezoidal fuzzy number can be represented as an ordered pair, i.e., $[(b-a) r+a,-(d-c) r+d]$, where $r \in[0,1]$.

\subsection{Triangular Fuzzy Number (TFN)}

Let $B=(a, b, c), a<b<c$, be a fuzzy set on $R=$ $(-\infty, \infty)$. It is called a triangular fuzzy number, if its membership function is

$$
\mu B(x)= \begin{cases}\frac{x-a}{b-a}, & \text { if } a \leq x \leq b \\ \frac{c-x}{c-b}, & \text { if } b \leq x \leq c \\ 0, & \text { otherwise }\end{cases}
$$

\section{Fractional order Improved Euler's method}

Caputo fractional derivatives implicate in generalization of Taylor's formula. The generalized fractional Euler's method that has been developed for evaluating the numerical solution of initial value problems with Caputo derivatives is presented by Odibat and Momani [28].

Suppose that $D_{x}^{k \alpha} f(x) \in C(0, a]$, for $k=0,1,2, \ldots, n+1$, where $0<\alpha \leq 1$

Then we have

$f(x)=\sum_{i=o}^{n} \frac{x^{i \alpha}}{\Gamma(i \alpha+1)}\left(D^{i \alpha}\right)(0+)+\frac{\left(D^{(n+1) \alpha} f\right)(\zeta)}{\Gamma((n+1) \alpha+1)} x^{(n+1) \alpha}$,

With $0 \leq \zeta \leq x, \forall x \in(0, a]$.

In case $\alpha=1$, of the generalized Taylor's formula, Eq. (13) reduces to the classical Taylor's formula. In order to find the high accuracy for solving fuzzy fractional differential equations, the initial value problem is considered as:

${ }^{c} D_{x}^{\alpha} \bar{y}_{n}(x)=f\left(x_{n}, \bar{y}_{n}\left(x_{n}\right)\right), \bar{y}(0)=\bar{y}_{0}, 0<\alpha \leq 1, \quad x_{n}>0$ 
The fuzzy fractional initial value problem (FFIVP) can be considered equivalent by the following initial value problems

$$
\left\{\begin{array}{c}
{ }^{c} D_{x}^{\alpha} \bar{y}_{n}^{r}(x)=\left[\bar{f}\left(x_{n}, \bar{y}_{n}\left(x_{n}\right)\right)\right]^{r}=F\left(x_{n}, \bar{y}_{n}^{r}\left(x_{n}\right), \underline{y}_{n}^{r}\left(x_{n}\right)\right) \\
{ }^{c} D_{x}^{\alpha} \underline{y}_{n}^{r}(x)=\left[\underline{f}\left(x_{n}, \bar{y}_{n}\left(x_{n}\right)\right)\right]^{r}=G\left(x_{n}, \bar{y}_{n}^{r}\left(x_{n}\right), \underline{y}_{n}^{r}\left(x_{n}\right)\right) \\
\bar{y}^{r}(0)=\bar{y}_{0}^{r}, \quad \underline{y}^{r}(0)=\underline{y}_{0}^{r}
\end{array}\right.
$$

Let $[0, a]$ be the interval over which we want to find the solution of the problem.

The focus is not to acquire a function $y(t)$ that satisfies the initial value problem. Alternately, an approximation has been made with the help of set of points $\left\{\left(x_{j}, y\left(x_{j}\right)\right)\right\}$.

$$
\begin{aligned}
\left(\bar{y}_{n+1}\left(x_{n+1}\right), \underline{y}_{n+1}\left(x_{n+1}\right)\right)= & \left(\bar{y}_{n}\left(x_{n}\right), \underline{y}_{n}\left(x_{n}\right)\right)+\frac{h^{\alpha}}{\Gamma(\alpha+1)}\left({ }^{c} D_{x}^{\alpha}\left(\bar{y}_{n}\left(x_{n}\right), \underline{y}_{n}\left(x_{n}\right)\right)\right)+\frac{h^{2 \alpha}}{\Gamma(2 \alpha+1)}\left({ }^{c} D_{x}^{2 \alpha}\left(\bar{y}_{n}\left(x_{n}\right), \underline{y}_{n}\left(x_{n}\right)\right)\right) \\
& +\frac{h^{3 \alpha}}{\Gamma(3 \alpha+1)}\left({ }^{c} D_{x}^{3 \alpha}\left(\bar{y}_{n}\left(x_{n}\right), \underline{y}_{n}\left(x_{n}\right)\right)\right)
\end{aligned}
$$

Since

$$
{ }^{c} D_{x}^{2 \alpha}\left(\bar{y}_{n}\left(x_{n}\right), \underline{y}_{n}\left(x_{n}\right)\right)=\Gamma(\alpha+1)\left(\frac{{ }^{c} D_{x}^{\alpha}\left(\bar{y}_{n+1}\left(x_{n+1}\right), \underline{y}_{n+1}\left(x_{n+1}\right)\right)-{ }^{c} D_{x}^{\alpha}\left(\bar{y}_{n}\left(x_{n}\right), \underline{y}_{n}\left(x_{n}\right)\right)}{h^{\alpha}}\right)+O\left(h^{\alpha}\right)
$$

Therefore

$$
\begin{gathered}
\left(\bar{y}_{n+1}\left(x_{n+1}\right), \underline{y}_{n+1}\left(x_{n+1}\right)\right)=\left(\bar{y}_{n}\left(x_{n}\right), \underline{y}_{n}\left(x_{n}\right)\right)+\frac{h^{\alpha}}{\Gamma(\alpha+1)}{ }^{c} D_{x}^{\alpha}\left(\bar{y}_{n}\left(x_{n}\right), \underline{y}_{n}\left(x_{n}\right)\right) \\
+\frac{h^{2 \alpha}}{\Gamma(2 \alpha+1)}\left(\Gamma(\alpha+1)\left(\frac{{ }^{c} D_{x}^{\alpha}\left(\bar{y}_{n+1}\left(x_{n+1}\right), \underline{y}_{n+1}\left(x_{n+1}\right)\right)-{ }^{c} D_{x}^{\alpha}\left(\bar{y}_{n}\left(x_{n}\right), \underline{y}_{n}\left(x_{n}\right)\right)}{h^{\alpha}}\right)+O\left(h^{\alpha}\right)\right)+O\left(h^{3 \alpha}\right) \\
\left(\bar{y}_{n+1}\left(x_{n+1}\right), \underline{y}_{n+1}\left(x_{n+1}\right)\right)= \\
\left(\bar{y}_{n}\left(x_{n}\right), \underline{y}_{n}\left(x_{n}\right)\right)+h^{\alpha}\left(\frac{\Gamma(2 \alpha+1)-(\Gamma(\alpha+1))^{2}}{(\Gamma(\alpha+1))(\Gamma(2 \alpha+1))}\right){ }^{c} D_{x}^{\alpha}\left(\bar{y}_{n}\left(x_{n}\right), \underline{y}_{n}\left(x_{n}\right)\right) \\
+\frac{h^{\alpha}(\Gamma(\alpha+1))}{\Gamma(2 \alpha+1)}{ }^{c} D_{x}^{\alpha}\left(\bar{y}_{n+1}\left(x_{n+1}\right), \underline{y}_{n+1}\left(x_{n+1}\right)\right)+O\left(h^{3 \alpha}\right)
\end{gathered}
$$

When

$$
{ }^{c} D_{x}^{\alpha} \bar{y}_{n}\left(x_{n}\right)=\bar{f}\left(x_{n}, y_{n}\left(x_{n}\right)\right), \quad{ }^{c} D_{x}^{\alpha} \underline{y}_{n}\left(x_{n}\right)=\underline{f}\left(x_{n}, y_{n}\left(x_{n}\right)\right), \quad{ }^{c} D_{x}^{\alpha} \bar{y}_{n+1}\left(x_{n+1}\right)=\bar{f}\left(x_{n+1}, y_{n+1}\left(x_{n+1}\right)\right)
$$

and ${ }^{c} D_{x}^{\alpha} \underline{y}_{n+1}\left(x_{n+1}\right)=\underline{f}\left(x_{n+1}, y_{n+1}\left(x_{n+1}\right)\right)$ are substituted into equation (18) the result is an expression for $y_{n+1}$ :

$$
\begin{aligned}
\left(\bar{y}_{n+1}\left(x_{n+1}\right), \underline{y}_{n+1}\left(x_{n+1}\right)\right)= & \left(\bar{y}_{n}\left(x_{n}\right), \underline{y}_{n}\left(x_{n}\right)\right)+h^{\alpha}\left(\frac{\Gamma(2 \alpha+1)-(\Gamma(\alpha+1))^{2}}{(\Gamma(\alpha+1))(\Gamma(2 \alpha+1))}\right)\left(\bar{f}\left(x_{n}, y_{n}\left(x_{n}\right)\right), \underline{f}\left(x_{n}, y_{n}\left(x_{n}\right)\right)\right) \\
& +\frac{h^{\alpha}(\Gamma(\alpha+1))}{\Gamma(2 \alpha+1)}\left(\bar{f}\left(x_{n+1}, y_{n+1}\left(x_{n+1}\right)\right), \underline{f}\left(x_{n+1}, y_{n+1}\left(x_{n+1}\right)\right)\right)
\end{aligned}
$$

A system of points that approximates the solution of $y(x)$ is produced by above recursive process. At each step, the fractional Euler's method is used as a prediction, and then the modified trapezoidal rule is used to make a correction to obtain the finite value. The general formula for the improved fractional Euler's algorithm is as follows:

$$
\begin{aligned}
y\left(x_{j}\right)= & \frac{h^{\alpha}}{\Gamma(\alpha+2)}\left(\begin{array}{l}
(j-1)^{\alpha+1} \\
-(j-\alpha-1) j^{\alpha}
\end{array}\right) f\left(x_{0}, y\left(x_{0}\right)\right)+y(0) \\
& +\frac{h^{\alpha}}{\Gamma(\alpha+2)} \sum_{i=0}^{j-1}\left((j-i+1)^{\alpha+1}-2(j-i)^{\alpha+1}+(j-i-1)^{\alpha+1}\right) f\left(x_{i}, y\left(x_{i}\right)\right) \\
& +\frac{h^{\alpha}}{\Gamma(\alpha+2)} f\left(x_{j}, y\left(x_{j-1}\right)+\frac{h^{\alpha}}{\Gamma(\alpha+1)} f\left(x_{j-1}, y\left(x_{j-1}\right)\right)\right) .
\end{aligned}
$$




\section{Basic idea of the MHPM}

To illustrate the basic idea of this method, let us consider the following nonlinear differential equation as described in [14]

$$
A(y(x))-f(r(x))=0 \quad x(x) \in \Omega
$$

with the following boundary conditions

$$
B(y(x), \partial y(x) / \partial n)=0 \quad r(x) \in \Gamma
$$

where $A$ is a general differential operator, $B$ is a boundary operator, $f(r(x))$ is a known analytical function and $\Gamma$ is the boundary of the domain $\Omega$. The operator $A$ can be divided into two parts, $L$ and $N$, where $L$ is a linear and $N$ is a nonlinear operator. Therefore Eq. (21) can be rewritten as

$$
L(y(x))-N(y(x))-f(r(x))=0
$$

By homotopy technique, construct a homotopy $U(r(x), p): \Omega \times[0,1] \rightarrow R$ which satisfies

$$
\begin{aligned}
H(Y(x), p)= & (1-p)\left[L\left(Y(x)-y_{0}(x)\right)\right] \\
& +p[A(Y(x))-f(r(x))]=0 \\
p & \in[0,1] r(x) \in \Omega
\end{aligned}
$$

or equivalently,

$$
\begin{aligned}
H(Y(x), p)= & \left(L(Y(x))-L\left(y_{0}(x)\right)\right)+p L\left(y_{0}(x)\right) \\
& +p[N(Y(x))-f(r(x))]=0
\end{aligned}
$$

where $p \in[0,1]$ is an embedding parameter, $y_{0}(x)$ is an initial approximation of solution of Eq. (21). Clearly, we have from Eq. (24) and (25).

$$
\begin{gathered}
H(Y(x), 0)=L(Y(x))-L\left(y_{0}(x)\right)=0 \\
H(Y(x), 1)=A(Y(x))-f(r(x))=0
\end{gathered}
$$

According to the MHPM, we can first use the embedding parameter $p$ as a small parameter, and assume that the solutions of equations (24) and (25) can be represented as a power series in $p$ as

$$
Y(x)=\sum_{n=0}^{\infty} p^{n} Y_{n}
$$

Now let us write the Eq. (25) in the following form

$$
L(Y(x))=y_{0}(x)+p\left[f(r(x))-y_{0}(x)-N(Y(x))\right]
$$

By applying the inverse operator, $L^{-1}$ to both sides of Eq. (29), we have

$$
\begin{aligned}
Y(x)= & L^{-1}\left(y_{0}(x)\right) \\
& +p\left[L^{-1}(f(r(x)))-L^{-1}\left(y_{0}(x)\right)-L^{-1}(N(Y(x)))\right]
\end{aligned}
$$

Suppose that the initial approximation of Eq. (23) has the form

$$
y_{0}(x)=\sum_{n=0}^{\infty} a_{n} P_{n}(x)
$$

where $a_{0}, a_{1}, a_{2}, \ldots$ are unknown coefficients and $P_{0}(x), P_{1}(x), P_{2}(x), \ldots$ are specific functions depending on the problem. Now by substituting Eq. (28) and Eq. (31) into the Eq. (30), we have

$$
\begin{aligned}
& \sum_{n=0}^{\infty} p^{n}(x) Y_{n}(x)=L^{-1}\left(\sum_{n=0}^{\infty} a_{n} P_{n}(x)\right) \\
& +p\left[L^{-1}(f(r(x)))-L^{-1}\left(\sum_{n=0}^{\infty} a_{n} P_{n}(x)\right)\right. \\
& \left.-L^{-1}\left(N\left(\sum_{n=0}^{\infty} p^{n} Y_{n}\right)\right)\right]
\end{aligned}
$$

Comparing coefficients of terms with identical powers of $p$, leads to

$$
\begin{gathered}
p^{0}: Y_{0}(x)=L^{-1}\left(\sum_{n=0}^{\infty} a_{n} P_{n}(x)\right), \\
p^{1}: Y_{1}(x)=L^{-1}(f(r(x)))-L^{-1}\left(\sum_{n=0}^{\infty} a_{n} P_{n}(x)\right) \\
-L^{-1}\left(N\left(Y_{0}(x)\right)\right),
\end{gathered}
$$

Now if we solve these equations in such a way that $Y_{1}(x)=0$, then Eq. (33) results in $Y_{2}(x)=Y_{3}(x)=\ldots=0$. Therefore, the exact solution may be obtained as following.

$$
y(x)=Y_{0}(x)=L^{-1}\left(\sum_{n=0}^{\infty} a_{n} P_{n}(x)\right) .
$$

It is worthwhile to mention that if $f(r(t))$ and $x_{0}(t)$ are analytic at $t=t_{0}$ then their Taylor series is defined as

$$
y_{0}(x)=\sum_{n=0}^{\infty} a_{n}\left(x-x_{0}\right)^{n}, f(r(x))=\sum_{n=0}^{\infty}\left(a_{n}^{*}\left(x-x_{0}\right)^{n}\right),
$$

can be used in Eq. (32), where $a_{0}^{\star}, a_{1}^{\star}, a_{2}^{\star}, \ldots$ are known coefficients and $a_{0}, a_{1}, a_{2}, \ldots$ are unknown ones, which must be computed.

To show the capability of this method, we apply the MHPM of fractional order to linear fuzzy initial value problems in the following section. 
Table 1. Solutions obtained by exact method, Duraisamy and Usha [25], MHPM and IFEM for $h=0.1$ at $t=1$.

\begin{tabular}{ccccc}
\hline $\mathbf{r}$ & Exact value & Duraisamy and Usha [25] & Improved Fractional Euler method $\boldsymbol{\alpha}=\mathbf{1}$ & MHPM(For Fractional Order) $\boldsymbol{\alpha}=\mathbf{1}$ \\
\hline 0 & {$[2.0387,3.0581]$} & {$[2.0355,3.0532]$} & {$[2.0356,3.0533]$} & {$[2.03871,3.05807]$} \\
0.2 & {$[2.1746,2.9901]$} & {$[2.1712,2.9853]$} & {$[2.1713,2.9855]$} & {$[2.17463,2.99011]$} \\
0.4 & {$[2.3105,2.9222]$} & {$[2.3070,2.9178]$} & {$[2.3070,2.9176]$} & {$[2.31054,2.92215]$} \\
0.6 & {$[2.4465,2.8542]$} & {$[2.4427,2.8496]$} & {$[2.4427,2.8498]$} & {$[2.44645,2.85420]$} \\
0.8 & {$[2.5824,2.7862]$} & {$[2.5784,2.7818]$} & {$[2.5784,2.7819]$} & {$[2.58237,2.78624]$} \\
1 & {$[2.7183,2.7183]$} & {$[2.7140,2.7140]$} & {$[2.7141,2.7141]$} & {$[2.71828,2.71828]$} \\
\hline
\end{tabular}

Table 2. Solutions obtained by MHPM and IFEM for $h=0.01$ at $t=0.1$ for different values of $\alpha$.

\begin{tabular}{ccccc}
\hline $\mathbf{r}$ & $\begin{array}{c}\text { MHPM(For Fractional Order) } \\
\boldsymbol{\alpha}=\mathbf{0 . 7 5}\end{array}$ & $\begin{array}{c}\text { Improved Fractional Euler method } \\
\boldsymbol{\alpha}=\mathbf{0 . 7 5}\end{array}$ & $\begin{array}{c}\text { Improved Fractional Euler method } \\
\boldsymbol{\alpha}=\mathbf{0 . 5}\end{array}$ & $\begin{array}{c}\text { MHPM(For Fractional Order) } \\
\boldsymbol{\alpha}=\mathbf{0 . 5}\end{array}$ \\
\hline 0 & {$[0.91369,1.37054]$} & {$[0.935716,1.40357]$} & {$[1.10577,1.65865]$} & {$[1.09650,1.64475]$} \\
0.2 & {$[0.97460,1.34008]$} & {$[0.998097,1.37238]$} & {$[1.17984,1.62179]$} & {$[1.16960,1.60820]$} \\
0.4 & {$[1.03552,1.30963]$} & {$[1.06048,1.34119]$} & {$[1.25320,1.58493]$} & {$[1.24270,1.57165]$} \\
0.6 & {$[1.09643,1.27917]$} & {$[1.12286,1.31000]$} & {$[1.32692,1.54807]$} & {$[1.31580,1.53510]$} \\
0.8 & {$[1.15734,1.24871]$} & {$[1.18524,1.27881]$} & {$[1.40064,1.51121]$} & {$[1.38890,1.49855]$} \\
1 & {$[1.21826,1.21826]$} & {$[1.24762,1.24762]$} & {$[1.47435,1.47435]$} & {$[1.46200,1.46200]$} \\
\hline
\end{tabular}

\section{Numerical problems}

\section{Example 1}

Let us consider a linear triangular FIVP given in [13] for fractional order.

$$
D_{t}^{\alpha} x(t)=x(t), \quad x(0)=(0.75,1,1.25 r), \quad 0 \leq r \leq 1 .
$$

Then using the $r$-cut approach, the triangular fuzzy initial condition can be represented as

$$
x(0)=[0.75+0.25 r, 1.125-0.125 r], \quad 0 \leq r \leq 1 .
$$

The results obtained by the above methods are tabulated in Table 1 and 2 for values of $h=0.1,0.01$, and $t=1,0.1$, respectively. In Table 1 , the exact solution is obtained for $t=1$ and the same is solved by the MHPM and by IFEM (for $\alpha=1$ ) and compared with known results of Duraisamy and Usha [25]. Table 2 presents the calculated values of considered problem for fractional order $\alpha=0.75,0.5$ from the methods MHPM, IFEM and compared.

\section{Example 2}

Let us consider now an initial value problem with initial trapezoidal fuzzy number with fractional order as below

$$
\begin{aligned}
& D_{t}^{\alpha} x(t)=x(t), \\
& x(0)=(0.75,0.85,1.1,1.25 r), 0 \leq r \leq 1 .
\end{aligned}
$$

Using $r$-cut, the initial trapezoidal fuzzy number may be written as

$$
x(0)=[0.75+0.1 r, 1.125-0.025 r], \quad 0 \leq r \leq 1 .
$$

In this example, the FFIVP with trapezoidal fuzzy initial condition is solved by the proposed method of MHPM. Corresponding results are given in Table 3 and 4 for values of $h=0.1,0.01$, and $t=1,0.1$, respectively. Table 3 provides the comparison of proposed methods with the exact solution and with the known results of Duraisamy and Usha [25] for $t=1$. The calculated values of trapezoidal problem for fractional order $\alpha=0.75,0.5$ from the methods MHPM, IFEM are presented and compared in Table 4. Example 3

Consider the nonlinear fuzzy fractional initial value problem as:

$$
D_{t}^{\alpha} x(t)=-\chi(t)+\chi^{2}(t), \quad \chi(0)=(0.75,1,1.5) .
$$

Using $r$-cut triangular fuzzy initial condition becomes

$$
x(0)=[0.75+0.25 r, 1.5-0.5 r], \quad 0 \leq r \leq 1 .
$$

The above nonlinear FFIVP (with TFN) is solved by the MHPM and IFEM for $h=0.1,0.001$ and computed results are shown in Table 5 and 6 at $t=1,0.1$, respectively. The comparisons of proposed methods with the Rung-Kutta method for $t=1$ are depicted in Table 5. The calculated values for fractional order $\alpha=0.75,0.5$ are presented and compared in Table 6 for the methods MHPM and IFEM.

\section{Conclusions}

The improved fractional Euler's method and two component homotopy perturbation method (MHPM) are successfully utilized to approximate the solutions for solving FIVP 
Table 3. Solutions obtained by exact method, Duraisamy and Usha [25], MHPM and IFEM for $h=0.1$ at $t=1$.

\begin{tabular}{ccccc}
\hline $\mathbf{r}$ & Exact value & Duraisamy and Usha [25] & Improved Fractional Euler method $\boldsymbol{\alpha}=\mathbf{1}$ & MHPM(For Fractional Order) $\boldsymbol{\alpha}=\mathbf{1}$ \\
\hline 0 & {$[2.0390,3.0580]$} & {$[2.0355,3.0532]$} & {$[2.0356,3.0533]$} & {$[2.03906,3.05807]$} \\
0.2 & {$[2.0934,3.0444]$} & {$[2.0898,3.0399]$} & {$[2.0898,3.0398]$} & {$[2.09308,3.04448]$} \\
0.4 & {$[2.1477,3.0308]$} & {$[2.1443,3.0266]$} & {$[2.1441,3.0262]$} & {$[2.14744,3.03088]$} \\
0.6 & {$[2.2021,3.0172]$} & {$[2.1985,3.0129]$} & {$[2.1984,3.0126]$} & {$[2.20181,3.01729]$} \\
0.8 & {$[2.2564,3.0036]$} & {$[2.2528,2.9990]$} & {$[2.2527,2.9991]$} & {$[2.25617,3.00370]$} \\
1 & {$[2.3108,2.9900]$} & {$[2.3070,2.9853]$} & {$[2.3070,2.9855]$} & {$[2.31054,2.99011]$} \\
\hline
\end{tabular}

Table 4. Solutions obtained by MHPM and IFEM for $h=0.01$ at $t=0.1$ For different values of $\alpha$.

\begin{tabular}{ccccc}
\hline $\mathbf{r}$ & MHPM(For Fractional Order) & Improved Fractional Euler method & $\begin{array}{c}\text { MHPM(For Fractional Order) } \\
\boldsymbol{\alpha}=\mathbf{0 . 5}\end{array}$ & $\begin{array}{c}\text { Improved Fractional Euler method } \\
\boldsymbol{\alpha}=\mathbf{0 . 7 5}\end{array}$ \\
\hline 0 & {$[0.903718,1.37054]$} & {$[0.93571,1.40357]$} & {$[1.03619,1.64475]$} & {$[1.10577,1.65865]$} \\
0.2 & {$[0.927818,1.36445]$} & {$[0.96066,1.39734]$} & {$[1.06383,1.63744]$} & {$[1.13525,1.65128]$} \\
0.4 & {$[0.951917,1.35836]$} & {$[0.98562,1.39110]$} & {$[1.09146,1.63013]$} & {$[1.16474,1.64391]$} \\
0.6 & {$[0.976016,1.35226]$} & {$[0.01057,1.38486]$} & {$[1.11909,1.62282]$} & {$[1.19423,1.63653]$} \\
0.8 & {$[1.00012,1.34617]$} & {$[1.03553,1.37862]$} & {$[1.14672,1.61551]$} & {$[1.22371,1.62916]$} \\
1 & {$[1.00421,1.34008]$} & {$[1.06048,1.37238]$} & {$[1.17435,1.60820]$} & {$[1.2532,1.62179]$} \\
\hline
\end{tabular}

Table 5. Solutions obtained by Runge kutta method, MHPM and IFEM for $h=0.1$ at $t=1$.

\begin{tabular}{cccc}
\hline $\mathbf{r}$ & Runge-Kutta Method & Improved Fractional Euler method $\boldsymbol{\alpha}=\mathbf{1}$ & MHPM(For Fractional Order) $\boldsymbol{\alpha}=\mathbf{1}$ \\
\hline 0 & {$[0.524633,10.6490]$} & {$[0.559054,8.64681]$} & {$[0.524633,7.36847]$} \\
0.2 & {$[0.595390,4.47732]$} & {$[0.595538,4.28938]$} & {$[0.595391,4.21255]$} \\
0.4 & {$[0.675814,2.68309]$} & {$[0.675979,2.65111]$} & {$[0.675814,2.66139]$} \\
0.6 & {$[0.768031,1.82831]$} & {$[0.768199,1.82190]$} & {$[0.768031,1.82716]$} \\
0.8 & {$[0.874839,1.32823]$} & {$[0.874971,1.32711]$} & {$[0.874839,1.32821]$} \\
1 & {$[1.00000,1.000000]$} & {$[1.00000,1.00000]$} & {$[1.000000,1.00000]$} \\
\hline
\end{tabular}

Table 6. Solutions obtained by MHPM and IFEM for $h=0.001$ at $t=0.1$. For different values of $\alpha$.

\begin{tabular}{ccccc}
\hline $\mathbf{r}$ & MHPM(For Fractional Order) & Improved Fractional Euler method & MHPM(For Fractional Order) & Improved Fractional Euler method \\
& $\boldsymbol{\alpha}=\mathbf{0 . 7 5}$ & $\boldsymbol{\alpha}=\mathbf{0 . 7 5}$ & $\boldsymbol{\alpha}=\mathbf{0 . 5}$ & $\boldsymbol{\alpha}=\mathbf{0 . 5}$ \\
\hline 0 & {$[0.71269,1.66354]$} & {$[0.76746,1.5352]$} & {$[0.68089,1.80768]$} & {$[0.83858,1.68639]$} \\
0.2 & {$[0.76798,1.52057]$} & {$[0.81864,1.43282]$} & {$[0.74062,1.62636]$} & {$[0.89480,1.57276]$} \\
0.4 & {$[0.82433,1.38292]$} & {$[0.86981,1.33044]$} & {$[0.80236,1.45535]$} & {$[0.95106,1.45932]$} \\
0.6 & {$[0.88177 .25040]$} & {$[0.92099,1.22807]$} & {$[0.86613,1.29423]$} & {$[1.00736,1.34606]$} \\
0.8 & {$[0.94032,1.12282]$} & {$[0.97217,1.12571]$} & {$[0.93200,1.14257]$} & {$[1.06370,1.23298]$} \\
1 & {$[1.00000,1.00000]$} & {$[1.02335,1.02335]$} & {$[1.00000,1.00000]$} & {$[1.12008,1.12008]$} \\
\hline
\end{tabular}

for fractional case. The computations corresponding to the examples have been performed using Mathematica 9.0. The exact solutions are compared with solutions obtained by means of the MHPM, IFEM and other known results as a special case. It is concluded that although both methods, MHPM and IFEM, can be used to solve nth fuzzy differential equations, but IFEM being applicable only for small values of dependent variable, failed to give accurate approximation for large values. Contrastingly, MHPM gives more accurate approximation even for larger values of dependent variable. Therefore, MHPM approximates more accurately and efficiently. The proposed methods can be employed to solve various kinds of fractional order fuzzy differential equations as well as fractional order integro- differential equations where the history of the problem at various time instant is of worth interest.

\section{Authors' contributions}

The authors have equal contributions to each part of this article. All the authors read and approved the final manuscript.

\section{Competing interest}

The authors have no competing interest. 


\section{References}

[1] S. L. Chang, L. A. Zadeh, On fuzzy mapping and control. IEEE Trans. Systems Man Cybernet 2(1) (1972): 30-34

[2] D. Dubois, H. Prade, Towards fuzzy differential calculus part 3: Differentiation. Fuzzy Sets and Systems 8(3) (1982): 225-233

[3] M. Puri, D. Ralescu, Differential and fuzzy functions, J. Math. Anal. Appl. 91(1983):552-558

[4] O. Kaleva, Fuzzy differential equations, Fuzzy Sets and Systems, 24(3) (1987): 301-317

[5] O. Kaleva, The Cauchy problem for fuzzy differential equations, Fuzzy Sets and Systems 35(3) (1990):389-396

[6] S. Seikkala, On the fuzzy initial value problem, Fuzzy Sets and Systems 24(3) (1987): 319-330

[7] S. Abbasbandy, T. Allahviranloo, Numerical solutions of fuzzy differential equations by Taylor method, Computational Methods in Applied Mathematics 2(2) (2002): 113-124

[8] T. Allahviranloo, N. Ahmady, E. Ahmady, Erratum to "Numerical solution of fuzzy differential equation by predictor-corrector method", Information Sciences 178(6) (2008): 1780-1782

[9] T. Allahviranloo, E. Ahmady, N. Ahmady, Nth-order fuzzy linear differential equations, Information Sciences 178(5) (2008): 1309-1324

[10] M, Friedman, M. Ma, A. Kandel, Numerical solutions of fuzzy differential and integral equations, Fuzzy Sets and Systems 106(1) (1999): 35-48

[11] M. Ma, M. Friedman, A. Kandel, Numerical solutions of fuzzy differential equations, Fuzzy Sets and Systems 105(1) (1999): 133-138

[12] N. A. Khan, M. Jamil, A. Ara, N.U. Khan, On efficient method for system of fractional differential equations, Advances in Difference Equations 2011 (2011): Article ADE/303472.

[13] A.M.A. El-Sayed, I.L. El-Kalla, E.A.A. Ziada, Analytical and numerical solutions of multi-term nonlinear fractional orders differential equations Applied Numerical Mathematics, 60 (8) (2010): 788-797

[14] N. A. Khan, A. Ara, M. Jamil, An efficient approach for solving the Riccati equation with fractional orders, Comp. Math. Appl. 61 (2011): 2683-2689.

[15] H-F. Ding, Y-X. Zhang, New numerical methods for the Riesz space fractional partial differential equations, Comp. \& Math. Appl. 63 (7) (2012): 1135-1146

[16] N. A. Khan, A. Mahmood, A. Ara, Nadeem A. Khan, Analytical study of nonlinear fractional order Integro-differential equation: Revisit Volterra's population model, Int. J of Differential Eq. (2012): Article ID 845945

[17] A. Pedas, E. Tamme, Numerical solution of nonlinear fractional differential equations by spline collocation methods, J. Comput. App. Math. 255 (2014): 216-230

[18] J. Shokri, Numerical solution of fuzzy differential equations, Applied Mathematical Sciences 1(2007):2231-2246

[19] S. Tapaswini, S. Chakraverty, A New Approach to Fuzzy Initial Value Problem by Improved Euler Method, Fuzzy Inf. Eng. 3(2012):293-312

[20] Chun C. Integration using He's homotopy perturbation method, Chaos Solutions Fractals 34(2007):1130-1134

[21] J.H. He, Some asymptotic methods for strongly nonlinear equations. Int. J. Modern Phys. B 20(2006):1141-1199
[22] E. Yusufoglu, An improvement to homotopy perturbation method for solving system of linear equations. Comput. Math. Appl. 58(2009):2231-2235

[23] H. Aminikhah, M. Hemmatnezhad, An effective modification of the homotopy perturbation method for stiff systems of ordinary differential equations. Appl. Math. Lett. 24(2011):15021508

[24] M. Jafari, M.M. Hosseini, S.T. Mohyud-Din, M. Ghovatmand, Modified homotopy perturbation method for solving nonlinear PDAEs and its applications in nanoelectronics. Int. J. Nonlinear Sci. Numer. Simul. 11(2010):1047-1058

[25] C. Duraisamy, B. Usha, Another approach to solution of Fuzzy differential equations by modified Euler's method, Proceedings of the International Conference on Communication and Computational Intelligence-kongu Engineering College, Perundurai, Erode, TN, India: (2010):52-55

[26] H. Aminikhah, M. Hemmatnezhad, An efficient method for quadratic Riccati differential equation, Commun. Nonlinear Sci. Numer. Simul. 15(2010): 835-839

[27] I. Podlubny, Fractional Differential Equations, Academic Press, San Diego, CA, (1999)

[28] Z. Odibat, S. Momani, An algorithm for the numerical solution differential equations of fractional order, J. Appl. Math. \& Informatics, 26 (1-2) (2008):15-27 